\title{
Nontrivial Fixed Points and Screening in the Hierarchical Two-Dimensional Coulomb Gas
}

\author{
G. Benfatto \\ Dipartimento di Matematica, II Università di Roma \\ 00133 Roma, Italy \\ J. Renn* \\ Department of Physics, Boston University \\ Boston, MA 02215, USA
}

\begin{abstract}
We show the existence and asymptotic stability of two fixed points of the renormalization group transformation for the hierarchical twodimensional Coulomb gas in the sine-Gordon representation and temperatures slightly greater than the critical one. We prove also that the correlations at the fixed points decay as in the hierarchical massive scalar free field theory, that is as $d_{x y}^{-4}$. We argue that this is the natural definition of screening in the hierarchical approximation.
\end{abstract}

\section{Introduction}

In the last ten years the renormalization group ideas have been extensively applied to the two dimensional Coulomb gas of identical charges $\pm e$, in order to rigorously understand the so called Kosterlitz-Thouless phase transition [1].

*Associate of the Department of Physics, Harvard University. Supported by Gruppo Nazionale per la Fisica Matematica, CNR, Italy. 
For inverse temperatures $\beta$ larger than the critical one, $\beta_{c}$, and small activity $\lambda$, it has been proved that there is no screening $[2,3]$. This result is strictly related to the fact that, in the field theoretical representation of the model (the so called sine-Gordon representation), the effective potential goes to zero as the scale goes to infinity [4]. All these results are valid also in the hierarchical approximation of the model (see Sect. 2), where they are obtained much easier $[5,6]$.

For $\beta<\beta_{c}$ screening is generally expected to be found, but this property has been proved in the exact model only for $\beta$ very small [7]. However, in the case of the hierarchical approximation, a weak form of screening has been proved for $\beta \nmid \beta_{c}[6]$.

In this paper we study the hierarchical Coulomb gas in the sine-Gordon representation in the region $\beta_{\star} \beta_{c}$, by analyzing the renormalization group transformation $\mathbf{T}$ of the effective potential. We prove that $\mathbf{T}$ has two nontrivial asymptotic stable fixed points, which have the following screening property: the two-charges truncated correlations decay as $d_{x y}^{-4}$ as the (hierarchical) distance $d_{x y}$ goes to infinity, which is the decaying behaviour of the truncated correlations of the massive hierarchical scalar field. Our analysis also suggests that the effective potential for the hierarchical Coulomb gas on scale 1 is in the domain of attraction of one of the fixed points, if the activity is small enough, so that in this case as well screening should be observed.

The existence of the nontrivial fixed points was proved in [6] with a different technique, which does not use the sine-Gordon representation. The proof of [6] extends to more general models, but allows one to study only the simple (not truncated) correlations. This is why the analysis of [6] was restricted to the screening for fractional charges; in fact, for the fractional charges, the truncated correlations coincide with the simple ones.

Finally, we want to stress that the technique used in this paper is essentially based on the bound discussed in the Appendix, which is a bound for the Ursell coefficients of a system of arbitrary charges sitting in the same point and interacting with a potential $c Q_{i} Q_{j}$. We were unable to find this estimate, which we think is interesting by itself, in the literature. 


\section{The hierarchical model}

Let $Q_{j}, j \in \mathbb{N}$, be a sequence of compatible pavements of $\mathbb{R}^{2}$ made of squares of side size $\gamma^{j}$, where $\gamma \geq 2$ is an integer. To each $\Delta \in Q_{j}$ we associate a gaussian variable $z_{\Delta}$ such that

$$
\mathcal{E}\left(z_{\Delta}^{2}\right)=\frac{1}{2 \pi} \log \gamma \quad, \quad \mathcal{E}\left(z_{\Delta} z_{\Delta^{\prime}}\right)=0 \text { if } \Delta \neq \Delta^{\prime}
$$

Then we define, $\forall x \in \mathbb{R}^{2}$ :

$$
\varphi_{x}=\sum_{k=0}^{\infty} z_{\Delta_{x}^{(k)}}
$$

where $\Delta_{x}^{(k)}$ is the tessera of side size $\gamma^{k}$ containing $x$.

Given $x, y \in \mathbb{R}^{2}$, let $h_{x y}$ be the smallest integer such that there exists a $\Delta \in Q_{h_{x y}}$ containing both $x$ and $y$. We shall call $d_{x y} \equiv \gamma^{h_{x y}}$, the side size of $\Delta$, the hierarchical distance between $x$ and $y$. By using (2.1) it is easy to see that:

$$
\mathcal{E}\left(\left(\varphi_{x}-\varphi_{y}\right)^{2}\right)=\mathcal{E}\left(\sum_{k=0}^{h_{x y}-1}\left[z_{\Delta_{x}^{(k)}}-z_{\Delta_{y}^{(k)}}\right]^{2}\right)=\frac{1}{\pi} \log d_{x y}
$$

which justifies the claim that $\varphi_{x}$ is a reasonable approximation of the twodimensional zero mass scalar field. In the following we shall denote the corresponding Gaussian measure by $P(d \varphi)$.

If $v(z), z \in \mathbb{R}^{1}$, is a real function such that

$$
v(0)=0 \quad, \quad v(z)=v(-z)
$$

and $\Lambda$ is a finite volume belonging to $Q_{R}$, for some $R>0$, we shall consider the measure

$$
\begin{aligned}
\mu_{v}^{\Lambda}(d \varphi) & =\frac{1}{Z_{v}^{\Lambda}} P(d \varphi) \prod_{\Delta \in Q_{0} \cap \Lambda} e^{v\left(\varphi_{\Delta}\right)} \\
Z_{v}^{\Lambda} & =\int P(d \varphi) \prod_{\Delta \in Q_{0} \cap \Lambda} e^{v\left(\varphi_{\Delta}\right)}
\end{aligned}
$$

where $\varphi_{\Delta}$ is the constant value of the field on the tessera $\Delta$.

The choice

$$
v(\varphi)=\lambda(\cos (\alpha \varphi)-1)
$$


corresponds to the hierarchical Coulomb gas in the volume $\Lambda$ with activity $\lambda / 2$, charges $\pm e$ and temperature $\beta^{-1}$, such that

$$
\beta e^{2}=\alpha^{2}
$$

For more details on this point see [5], where a rescaled field was used, instead of $(2.2)$.

Another interesting choice is

$$
v(\varphi)=-m^{2} \varphi^{2} \equiv u_{m}(\varphi)
$$

which should give rise to the two dimensional hierarchical scalar field of mass $m$. It is important to remark, however, that this is not a good approximation of the massive scalar field. In fact it is easy to show that

$$
\lim _{\Lambda \rightarrow \mathbb{R}^{2}} \int \mu_{u_{m}}^{\Lambda}(d \varphi) \varphi_{x} \varphi_{y} \propto d_{x y}^{-4}
$$

in disagreement with the exponential decay of the massive scalar field (a similar property is valid in other hierarchical models, see [8], chapter 4, exercise 2).

This observation will be very relevant in the following, since it implies that the hierarchical Coulomb gas should have power decaying correlations also in a screened phase, but with a power equal to 4 independently of $\beta$.

Let us now define the renormalization group transformation.

If $F(z)$ is a function on $\mathbb{R}$ and $\left\langle\cdot>_{v}^{\Lambda}\right.$ denotes the expectation w.r.t. the measure (2.5), then

$$
<F\left(\varphi_{0}\right)>_{v} \equiv \lim _{\Lambda \rightarrow \mathbb{R}^{2}}<F\left(\varphi_{0}\right)>_{v}^{\Lambda}=<\mathbf{L}_{\mathbf{T}^{k-1} v} \cdots \mathbf{L}_{v} F\left(\varphi_{0}\right)>_{\mathbf{T}^{k} v}
$$

where

$$
\begin{aligned}
(\mathbf{T} v)(\varphi) & =\log \left[\frac{\int P_{0}(d z) e^{v(\varphi+z)}}{\int P_{0}(d z) e^{v(z)}}\right]^{\gamma^{2}} \\
\left(\mathbf{L}_{v} F\right)(\varphi) & =\frac{\int P_{0}(d z) e^{v(\varphi+z)} F(\varphi+z)}{\int P_{0}(d z) e^{v(\varphi+z)}}
\end{aligned}
$$

if $P_{0}(d z)$ denotes the gaussian measure on $\mathbb{R}^{1}$ of mean zero and covariance $\frac{1}{2 \pi} \log \gamma$. 
The operators $\mathbf{T}$ and $\mathbf{L}_{v}$ appear also in the expressions similar to (2.11) valid for the expectations of any observable depending on the values of the field $\varphi_{x}$ in a finite set of tesserae $\Delta \in Q_{0}$.

The operator (2.12) is the renormalization group transformation. It leaves invariant the space $\mathfrak{C}_{\alpha}$ of the continuous functions periodic of period $T_{\alpha}=$ $2 \pi / \alpha$ and satisfying (2.4); then, if we want to study the hierarchical Coulomb gas at temperature $\beta^{-1}$, we have to restrict $\mathbf{T}$ to $\mathfrak{C}_{\alpha}$ with $\alpha=\sqrt{\beta e^{2}}$. In Ref. [5] it was implicitly shown that $v_{0}(\varphi)=0$ is, for $\alpha^{2}>8 \pi$, a fixed point of (2.12) which is attracting for functions of the form (2.7), for $\lambda$ small enough. In fact one could also show that it is locally attracting in some subspace of sufficiently regular functions.

In this paper we shall study the more difficult case $\alpha^{2} \leq 8 \pi$ and we shall prove that there are two stable fixed points $v_{\alpha}(\varphi) \neq 0$ (this result, as discussed in the Introduction, has already been obtained with a different technique [6]) in a suitable subspace of $\mathfrak{C}_{\alpha}$.

Moreover, by studying the spectrum of (2.13) for $v=v_{\alpha}$ in the space of $L_{2}$ functions periodic of period $T_{\alpha}$, we shall prove that the integer charge truncated correlations decay like $d_{x y}^{-4}$. The restriction of $\mathbf{L}_{v}$ to periodic functions is motivated by the fact that the truncated integer charge correlations are given by the formula

$$
\rho^{T}\left(x_{1}, \sigma_{1} ; \ldots ; x_{n}, \sigma_{n}\right)=\frac{\partial^{n}}{\partial \omega_{1} \cdots \partial \omega_{n}} \log <\exp \left\{\frac{\lambda}{2} \sum_{j=1}^{n} \omega_{j} e^{i \alpha \sigma_{j} \varphi_{x_{j}}}\right\}>\left.\right|_{\omega_{j}=0}
$$

where $\sigma_{i} \in\{-1,1\}$ are the charges and $x_{i}$ are the positions of $n$ particles.

\section{The existence of two attracting nontrivial fixed points}

The proof of the existence of nontrivial fixed points for $\alpha^{2}<8 \pi$ is based on the perturbative expansion of the renormalization group transformation (2.12). If $v(\varphi) \in \mathfrak{C}_{\alpha}$, we can write:

$$
v(\varphi)=\sum_{0 \neq Q \in \mathbb{Z}} v_{Q}\left(e^{i \alpha Q \varphi}-1\right)
$$


where $v_{Q}=v_{-Q}$. Then, if $v_{Q}^{\prime}$ are the Fourier coefficients of $(\mathbf{T} v)(\varphi),(2.12)$ can be written in the following form:

$$
v_{Q}^{\prime}=\gamma^{2-\frac{\alpha^{2}}{4 \pi} Q^{2}} v_{Q}+\gamma^{2} \sum_{n=2}^{\infty} \frac{1}{n !} \sum_{Q_{1}+\cdots+Q_{n}=Q} v_{Q_{1}} \cdots v_{Q_{n}} F_{n}\left(Q_{1}, \ldots, Q_{n}\right)
$$

where

$$
F_{n}\left(Q_{1}, \ldots, Q_{n}\right)=\mathcal{E}^{T}\left(e^{i \alpha Q_{1} z} ; \ldots ; e^{i \alpha Q_{n} z}\right)
$$

if we denote $\mathcal{E}^{T}$ the truncated expectation with respect to the measure $P_{0}(d z)$.

In the appendix we prove the following nontrivial bound, which will play a crucial role in the following:

$$
\left|F_{n}\left(Q_{1}, \ldots, Q_{n}\right)\right| \leq n^{n-1}\left[\prod_{i=1}^{n}\left(4 \sqrt{\kappa}\left|Q_{i}\right|\right)\right] e^{-\kappa \frac{Q^{2}}{n}}
$$

where $\kappa=\left(\alpha^{2} / 8 \pi\right) \log \gamma$ and $Q=\sum_{i=1}^{n} Q_{i}$.

The linearization of (3.2) around the fixed point $v=0$ has a bifurcation at $\alpha^{2}=8 \pi$, where the Fourier coefficients $v_{ \pm 1}$ become unstable. This bifurcation, as it is well known, is responsible for the Kosterlitz-Thouless phase transition. In this paper we study the range of temperatures, immediately above the critical one, given by the relation

$$
0<\varepsilon \equiv 2-\frac{\alpha^{2}}{4 \pi} \leq \varepsilon_{0}
$$

with $\varepsilon_{0}$ small enough. We start by proving that (3.2) has two fixed points different from zero.

We first look for approximate solutions, by imposing in (3.2) the conditions $|Q|,\left|Q_{i}\right| \leq 2$ and $n \leq 2$. Taking into account the symmetry property $v_{Q}=v_{-Q}$, we obtain a system of two equations:

$$
\begin{aligned}
& v_{1}^{\prime}=\gamma^{\varepsilon} v_{1}+a v_{1} v_{2}-f v_{1}^{3} \\
& v_{2}^{\prime}=c v_{2}-b v_{1}^{2}
\end{aligned}
$$

where

$$
\begin{aligned}
a & =\gamma^{2} F_{2}(-1,2)=\gamma^{\varepsilon}\left(1-\gamma^{-4(2-\varepsilon)}\right) \\
b & =-\frac{1}{2} \gamma^{2} F_{2}(1,1)=\frac{1}{2} \gamma^{-2(1-\varepsilon)}\left(1-\gamma^{-4+2 \varepsilon}\right) \\
c & =\gamma^{-6+4 \varepsilon} \\
f & =-\frac{1}{2} \gamma^{2} F_{3}(-1,1,1)=\frac{1}{2} \gamma^{\varepsilon}\left(1-\gamma^{-4+2 \varepsilon}\right)^{2}
\end{aligned}
$$


If $\varepsilon>0$ the system (3.7) has two solutions different from zero given by:

$$
\begin{aligned}
& \bar{v}_{1}^{2}=\frac{(1-c)\left(\gamma^{\varepsilon}-1\right)}{a b+f(1-c)} \\
& \bar{v}_{2}=\frac{-b\left(\gamma^{\varepsilon}-1\right)}{a b+f(1-c)}
\end{aligned}
$$

Furthermore it is possible to see that, if $\bar{v}_{1}^{+}$and $\bar{v}_{1}^{-}$are, respectively, the positive and the negative solution, there is a neighbourhood $\mathfrak{S}$ of the origin in $\mathbb{R}^{2}$, containing $\bar{v}_{1}^{+}$and $\bar{v}_{1}^{-}$, such that $\mathfrak{S} \cap\left\{v_{1}>0\right\}$ and $\mathfrak{S} \cap\left\{v_{1}<0\right\}$ are in the domain of attraction, respectively, of $\bar{v}_{1}^{+}$and $\bar{v}_{1}^{-}$.

In the following we shall consider only the solution with $\bar{v}_{1}>0$, but the same considerations could be applied to the other one. We want to prove that there is a fixed point of (2.12) which is approximately equal to the function

$$
\bar{v}(\varphi)=2 \bar{v}_{1}[\cos \varphi-1]+2 \bar{v}_{2}[\cos (2 \varphi)-1]
$$

We want to apply the contraction mapping principle; hence we need to define a suitable Banach space and find a $\mathbf{T}$-invariant subset, containing (3.9), on which $\mathbf{T}$ is a contraction with respect to a suitable metric.

Let us consider the functions of $\mathfrak{C}_{\alpha}$, which are analytic and bounded in a symmetric strip along the real axis of width $2 \tilde{b}$ such that

$$
\delta \equiv e^{-\alpha \tilde{b}} \equiv \bar{a} \varepsilon^{\frac{1}{2}} \leq \bar{\delta}<1
$$

These functions form a Banach space $\mathfrak{B}$, if we define the norm in the following way:

$$
\|v\|=\sup _{Q \geq 1} \delta^{-Q}\left|v_{Q}\right|
$$

Let $\mathfrak{B}_{d} \subset \mathfrak{B}$ be the sphere of radius $d$ with center at the origin. We want to choose $d$ and $\bar{a}$, see (3.10), so that the smaller sphere $\mathfrak{B}_{d / 2}$ contains the function $\bar{v}$, see (3.9). From (3.8) it follows that this is possible if

$$
\begin{aligned}
2 \sqrt{\frac{1-c}{a b+f(1-c)} \frac{\gamma^{\varepsilon}-1}{\varepsilon}} & \leq d \bar{a} \\
2 \frac{b}{a b+f(1-c)} \frac{\gamma^{\varepsilon}-1}{\varepsilon} & \leq d \bar{a}^{2}
\end{aligned}
$$


The bounds (3.12) and (3.10) can be satisfied for any $d$, if $\bar{a}$ is sufficiently large and $\varepsilon$ is sufficiently small, as we shall suppose in the following.

We want now to define a subset $\mathfrak{D} \subset \mathfrak{B}_{d}$ containing the functions which are close to $\bar{v}$. Let us define:

$$
v_{i}=\bar{v}_{i}+r_{i} \quad, \quad v_{i}^{\prime}=\bar{v}_{i}+r_{i}^{\prime} \quad, \quad i=1,2
$$

and consider the linear change of coordinates which diagonalizes the linearization of the system (3.6) around its fixed point, that is:

$$
\left(\begin{array}{l}
r_{1} \\
r_{2}
\end{array}\right)=S\left(\begin{array}{l}
u_{1} \\
u_{2}
\end{array}\right)
$$

where

$$
S=\left(\begin{array}{cc}
1 & -a \sigma \bar{v}_{1} \\
-2 b \sigma \bar{v}_{1} & 1
\end{array}\right)
$$

with

$$
\begin{aligned}
\sigma & =\frac{1}{1-c-K\left(\gamma^{\varepsilon}-1\right)} \\
K & =\frac{4(1-c)}{\left(1+2 f \bar{v}_{1}^{2}-c\right)\left[1+\sqrt{1-\frac{8(1-c)\left(\gamma^{\varepsilon}-1\right)}{\left(1+2 f \bar{v}_{1}^{2}-c\right)^{2}}}\right]}
\end{aligned}
$$

$\mathfrak{D}$ is the set of functions $v \in \mathfrak{B}_{d}$, such that:

$$
\begin{aligned}
& \left|u_{1}\right| \leq \tilde{d} \varepsilon^{1+\eta} \\
& \left|u_{2}\right| \leq \tilde{d} \varepsilon^{3 / 2}
\end{aligned}
$$

where $0<\eta<1 / 2$ and $\tilde{d}$ is any fixed positive constant.

Theorem 3.1 There exist a positive constant $d_{0}$ and, for any given $d, \tilde{d}, \eta$, such that $0<d \leq d_{0}$ and $0<\eta<1 / 2$, another constant $\varepsilon_{0}$, so that the set $\mathfrak{D}$ is invariant under the transformation $\mathbf{T}$, if $\varepsilon \leq \varepsilon_{0}$.

Proof - (3.2) and (3.4) imply that, if $v \in \mathfrak{B}_{d}$ and $Q \geq 1$ :

$$
\begin{aligned}
\left|v_{Q}^{\prime}\right| & \leq \gamma^{2-Q^{2}(2-\varepsilon)} d \delta^{Q}+\sum_{n=2}^{\infty}(B d \sqrt{\kappa})^{n} e^{-\kappa \frac{Q^{2}}{n}} \sum_{Q_{1}+\cdots+Q_{n}=Q} \prod_{i=1}^{n}\left|Q_{i}\right| \delta^{\left|Q_{i}\right|} \\
& \leq \gamma^{2-Q^{2}(2-\varepsilon)} d \delta^{Q}+\sum_{n=2}^{\infty}\left(B_{1} d \sqrt{\kappa}\right)^{n} e^{-\kappa \frac{Q^{2}}{n}} \sum_{\substack{Q_{1}+\cdots+Q_{n}=Q \\
\left|Q_{i}\right| \geq 1}} \prod_{i=1}^{n} \delta_{1}^{\left|Q_{i}\right|}
\end{aligned}
$$


where $B, B_{1}$ are suitable positive constants and $\delta_{1}$ is chosen so that

$$
\bar{\delta}<\delta_{1}<1
$$

We now have to carefully bound the sum:

$$
I \equiv \sum_{n=2}^{\infty}\left(B_{1} d \sqrt{\kappa}\right)^{n} e^{-\kappa \frac{Q^{2}}{n}} \sum_{\substack{Q_{1}+\cdots+Q_{n}=Q \\\left|Q_{i}\right| \geq 1}} \prod_{i=1}^{n} \delta_{1}^{\left|Q_{i}\right|}
$$

We can write:

$$
I=I_{0}+I_{1}
$$

with

$$
\begin{gathered}
I_{0}=\sum_{n=2}^{Q}\left(B_{1} d \sqrt{\kappa}\right)^{n} e^{-\kappa \frac{Q^{2}}{n}} \delta_{1}^{Q} N_{n}(Q) \\
I_{1}=\sum_{n=2}^{\infty}\left(B_{1} d \sqrt{\kappa}\right)^{n} e^{-\kappa \frac{Q^{2}}{n}} \sum_{k=1}^{n-1}\left(\begin{array}{c}
n \\
k
\end{array}\right) \sum_{s=k}^{\infty} \delta_{1}^{Q+2 s} N_{k}(s) N_{n-k}(Q+s)
\end{gathered}
$$

where the combinatorial factor $N_{k}(Q)$ is defined as

$$
N_{k}(Q)=\sum_{\substack{Q_{1}+\cdots+Q_{k}=Q \\
Q_{i} \geq 1}} 1=\left\{\begin{array}{cc}
\left(\begin{array}{c}
Q-1 \\
k-1
\end{array}\right) & \text { for } Q \geq k \geq 1 \\
0 & \text { for } 1 \leq Q<k
\end{array}\right.
$$

It is easy to see that

$$
I_{0} \leq \kappa Q e^{-\kappa Q}\left(B_{1} d\right)^{2}\left(1+B_{1} d \sqrt{\kappa}\right)^{Q-2} \delta_{1}^{Q}
$$

In order to bound $I_{1}$, we use the inequality

$$
\left(\begin{array}{c}
s \\
k
\end{array}\right) \leq \frac{s^{k}}{k !} \leq \frac{e^{\rho s}}{\rho^{k}}
$$

valid for any positive $\rho$ and we choose $\rho$ so that

$$
\delta_{2} \equiv \delta_{1} e^{\rho}<1
$$

Then we have:

$$
I_{1} \leq \delta_{2}^{Q}\left(\delta_{1} B_{1} d \sqrt{\kappa}\right)^{2} \sum_{n=2}^{\infty}\left(\frac{B_{1} d \sqrt{\kappa}}{\rho}\right)^{n-2} e^{-\kappa \frac{Q^{2}}{n}} \frac{\left(1+\delta_{2}^{2}\right)^{n}-1-\delta_{2}^{2 n}}{\delta_{2}^{2}\left(1-\delta_{2}^{2}\right)}
$$


If $d$ is sufficiently small, we have also:

$$
\frac{B_{1} d \sqrt{\kappa}}{\rho}\left(1+\delta_{2}^{2}\right) \leq \bar{\delta}<1
$$

which is compatible with (3.10) and (3.12) for $\varepsilon$ small enough and $\bar{a}$ large enough. (3.22), (3.28) and (3.29) easily imply that

$$
I \leq \bar{A}\left(B_{1} d\right)^{2} \delta^{Q}
$$

for a suitable positive constant $\bar{A}$, only depending on $\bar{\delta}$ and $\delta_{1}$.

The inequalities (3.18) and (3.30) imply that, if $Q \geq 3,\left|v_{Q}^{\prime}\right| \leq d \delta^{Q}$ provided that

$$
\gamma^{2-9(2-\varepsilon)} d+\bar{A}\left(B_{1} d\right)^{2} \leq d
$$

which can be satisfied if $d$ is sufficiently small. Then, in order to prove that $\mathfrak{D}$ is invariant, we still have to check that the condition (3.17) is preserved.

Let us notice that:

$$
\left(\begin{array}{c}
r_{1}^{\prime} \\
r_{2}^{\prime}
\end{array}\right)=\left(\begin{array}{cc}
1-2 f \bar{v}_{1}^{2} & a \bar{v}_{1} \\
-2 b \bar{v}_{1} & c
\end{array}\right)\left(\begin{array}{c}
r_{1} \\
r_{2}
\end{array}\right)+\left(\begin{array}{c}
\psi_{1} \\
\psi_{2}
\end{array}\right)+\left(\begin{array}{c}
\tilde{v}_{1} \\
\tilde{v}_{2}
\end{array}\right)
$$

where

$$
\begin{aligned}
& \psi_{1}=a r_{1} r_{2}-3 f \bar{v}_{1} r_{1}^{2}-f r_{1}^{3} \\
& \psi_{2}=-b r_{1}^{2}
\end{aligned}
$$

and

$$
\begin{aligned}
& \tilde{v}_{1}=\gamma^{2} \sum_{n=2}^{\infty} \frac{1}{n !} \sum_{\substack{Q_{1}+\cdots+Q_{n}=1 \\
\left|Q_{1}\right|+\cdots\left|Q_{n}\right| \geq 5}} v_{Q_{1}} \cdots v_{Q_{n}} F_{n}\left(Q_{1}, \ldots, Q_{n}\right) \\
& \tilde{v}_{2}=\gamma^{2} \sum_{n=2}^{\infty} \frac{1}{n !} \sum_{\substack{Q_{1}+\cdots+Q_{n}=2 \\
\left|Q_{1}\right|+\cdots\left|Q_{n}\right| \geq 4}} v_{Q_{1}} \cdots v_{Q_{n}} F_{n}\left(Q_{1}, \ldots, Q_{n}\right)
\end{aligned}
$$

Proceeding as before, it is easy to show that, if $d$ is sufficiently small, then

$$
\begin{aligned}
& \left|\tilde{v}_{1}\right| \leq d \delta^{5}=d \bar{a}^{5} \varepsilon^{5 / 2} \\
& \left|\tilde{v}_{2}\right| \leq d \delta^{4}=d \bar{a}^{4} \varepsilon^{2}
\end{aligned}
$$

The previous considerations imply that there exists $d_{0}>0$, such that, given $d \leq d_{0},(3.10),(3.12),(3.29),(3.31)$ and (3.35) are satisfied for $\varepsilon$ sufficiently small. 
By some simple algebra and using (3.14), it is possible to show that

$$
\left(\begin{array}{c}
u_{1}^{\prime} \\
u_{2}^{\prime}
\end{array}\right)=\left(\begin{array}{c}
\lambda_{1} u_{1} \\
\lambda_{2} u_{2}
\end{array}\right)+\left(\begin{array}{c}
R_{1} \\
R_{2}
\end{array}\right)+\left(\begin{array}{c}
\tilde{v}_{1}+a \tilde{\sigma} \bar{v}_{1} \tilde{v}_{2} \\
\tilde{v}_{2}+2 b \tilde{\sigma} \bar{v}_{1} \tilde{v}_{1}
\end{array}\right)
$$

where

$$
\begin{aligned}
& \lambda_{1}=1-K\left(\gamma^{\varepsilon}-1\right) \\
& \lambda_{2}=\gamma^{-6+4 \varepsilon}+K\left(\gamma^{\varepsilon}-1\right)-2 f \bar{v}_{1}^{2}
\end{aligned}
$$

and

$$
\begin{gathered}
\left(\begin{array}{c}
R_{1} \\
R_{2}
\end{array}\right)=\tilde{\sigma}\left(\begin{array}{c}
a r_{1} r_{2}-(3 f+a b \sigma) \bar{v}_{1} r_{1}^{2}-f r_{1}^{3} \\
-b r_{1}^{2}+2 b \tilde{\sigma} \bar{v}_{1}\left(a r_{1} r_{2}-3 f \bar{v}_{1} r_{1}^{2}-f r_{1}^{3}\right)
\end{array}\right) \\
\tilde{\sigma}=\frac{\sigma}{1-2 a b \sigma^{2} \bar{v}_{1}^{2}}
\end{gathered}
$$

By using (3.8), (3.14), (3.35), and (3.36), it is easy to prove that, if $0<\eta<1 / 2$, and $\varepsilon$ is sufficiently small, say $\varepsilon \leq \varepsilon_{0}$, then

$$
\begin{aligned}
& \left|u_{1}^{\prime}\right| \leq \lambda_{1} \tilde{d} \varepsilon^{1+\eta}+c_{1} \tilde{d}^{3} \varepsilon^{5 / 2+\eta}+c_{2} d \bar{a}^{5} \varepsilon^{5 / 2} \\
& \left|u_{2}^{\prime}\right| \leq \lambda_{2} \tilde{d} \varepsilon^{3 / 2}+c_{3} \tilde{d}^{3} \varepsilon^{2+2 \eta}+c_{4} d \bar{a}^{5} \varepsilon^{2}
\end{aligned}
$$

for suitable constants $c_{i}, i=1, \ldots, 4$. Then the conditions (3.17) are preserved if

$$
\begin{aligned}
& \lambda_{1}+c_{1} \tilde{d}^{2} \varepsilon^{3 / 2}+c_{2} \frac{d}{\tilde{d}} \bar{a}^{5} \varepsilon^{3 / 2-\eta} \leq 1 \\
& \lambda_{2}+c_{3} \tilde{d}^{2} \varepsilon^{1 / 2+2 \eta}+c_{4} \frac{d}{\tilde{d}} \bar{a}^{5} \varepsilon^{1 / 2} \leq 1
\end{aligned}
$$

By looking at (3.37), it is immediate to see that (3.41) can be satisfied, given any $0<\eta<1 / 2$, if $\varepsilon$ is small enough. In order to prove that $\mathbf{T} v \in \mathfrak{D}$, we still have to check that

$$
\left|v_{1}^{\prime}\right| \leq d \delta \quad, \quad\left|v_{2}^{\prime}\right| \leq d \delta^{2}
$$

which is again true for any $\eta<1 / 2$, if $\varepsilon$ is small enough, by (3.13), (3.17) and the fact that $\bar{v} \in \mathfrak{B}_{d / 2}$.

We now want to show that $\mathfrak{D}$ contains a fixed point $v^{*}$ of the transformation $\mathbf{T}$ and that, given any $v \in \mathfrak{D},\left\|\mathbf{T}^{n} v-v^{*}\right\| \rightarrow 0$ as $n \rightarrow \infty$. 
Given two elements of $\mathfrak{D}, v^{(1)}$ and $v^{(2)}$, we define $r_{i}^{(j)}=v_{i}^{(j)}-\bar{v}_{i}^{(j)}, j, i=$ 1,2 , and $u_{i}^{(j)}$ as in (3.13) and (3.14); then we define:

$$
\mathfrak{m}\left(v^{(1)}, v^{(2)}\right)=\max \left\{\delta^{-1}\left|u_{1}^{(1)}-u_{1}^{(2)}\right|, \delta^{-2}\left|u_{2}^{(1)}-u_{2}^{(2)}\right|, \sup _{Q \geq 3} \delta^{-Q}\left|v_{Q}^{(1)}-v_{Q}^{(2)}\right|\right\}
$$

It is easy to see that

$$
\begin{aligned}
& \left|v_{1}^{(1)}-v_{1}^{(2)}\right|=\left|r_{1}^{(1)}-r_{1}^{(2)}\right| \leq c_{5} \delta \mathfrak{m}\left(v^{(1)}, v^{(2)}\right) \\
& \left|v_{2}^{(1)}-v_{2}^{(2)}\right|=\left|r_{2}^{(1)}-r_{2}^{(2)}\right| \leq c_{5} \delta^{2} \mathfrak{m}\left(v^{(1)}, v^{(2)}\right)
\end{aligned}
$$

and

$$
\begin{aligned}
& \left|u_{1}^{(1)}-u_{1}^{(2)}\right| \leq c_{5} \delta\left\|v^{(1)}-v^{(2)}\right\| \\
& \left|u_{2}^{(1)}-u_{2}^{(2)}\right| \leq c_{5} \delta^{2}\left\|v^{(1)}-v^{(2)}\right\|
\end{aligned}
$$

for some constant $c_{5}$.

The inequalities (3.44) and (3.45) imply that $\mathfrak{m}$ and the norm (3.11) generate the same topology. Therefore, in order to prove the existence of a fixed point $v^{*}$ in $\mathfrak{D}$ and its asymptotic stability, the following theorem is sufficient.

Theorem 3.2 There exist a positive constant $d_{1} \leq d_{0}$ and, for any given $d \leq d_{1}$ and $0<\eta<1 / 2$, another constant $\varepsilon_{1} \leq \varepsilon_{0}$, such that, for any $\varepsilon \leq \varepsilon_{1}$ :

$$
\mathfrak{m}\left(\mathbf{T} v^{(1)}, \mathbf{T} v^{(2)}\right) \leq \nu_{\varepsilon} \mathfrak{m}\left(v^{(1)}, v^{(2)}\right)
$$

with $\nu_{\varepsilon}<1$ and $\nu_{\varepsilon} \rightarrow 1$ as $\varepsilon \rightarrow 0$.

Proof - By proceeding as in the proof of Theorem 3.1 and using the identity

$$
v_{Q_{1}}^{(1)} \cdots v_{Q_{n}}^{(1)}-v_{Q_{1}}^{(2)} \cdots v_{Q_{n}}^{(2)}=\sum_{k=1}^{n} v_{Q_{1}}^{(1)} \cdots v_{Q_{k-1}}^{(1)}\left[v_{Q_{k}}^{(1)}-v_{Q_{k}}^{(2)}\right] v_{Q_{k+1}}^{(2)} \cdots v_{Q_{n}}^{(2)}
$$

and (3.44), it is easy to show that, if $Q \geq 3$ and $d$ is sufficiently small, then

$$
\delta^{-Q}\left|\left(\mathbf{T} v^{(1)}\right)_{Q}-\left(\mathbf{T} v^{(2)}\right)_{Q}\right| \leq\left[\gamma^{2-9(2-\varepsilon)}+B_{2} d\right] \mathfrak{m}\left(v^{(1)}, v^{(2)}\right)
$$

for some constant $B_{2}$, depending only on $\bar{\delta}$ and $\delta_{1}$. 
Moreover, by using (3.36), it is easy to show that

$$
\begin{aligned}
\delta^{-1}\left|u_{1}^{\prime(1)}-u_{1}^{\prime(2)}\right| & \leq\left[\lambda_{1}+c_{6} \varepsilon^{3 / 2}+c_{6} \varepsilon^{2}\right] \mathfrak{m}\left(v^{(1)}, v^{(2)}\right) \\
\delta^{-2}\left|u_{2}^{\prime(1)}-u_{2}^{\prime(2)}\right| & \leq\left[\lambda_{2}+c_{6} \varepsilon^{1 / 2+\eta}+c_{6} \varepsilon\right] \mathfrak{m}\left(v^{(1)}, v^{(2)}\right)
\end{aligned}
$$

for $d$ small enough, say $d \leq d_{1} \leq d_{0}$, and some constant $c_{6}$ depending on $\tilde{d}, \bar{a}$ and $d_{1}$.

All the claims of the theorem easily follow.

Theorem 3.2 is not completely satisfactory, since we are interested in the properties of the measure $(2.5)$ with potential $v(\varphi)=\lambda(\cos \varphi-1) \equiv v^{(\lambda)}(\varphi)$. The properties of the approximate transformation (3.6) (see discussion after (3.8)) suggest that $v^{(\lambda)}$ is in the domain of attraction of $v^{*}$, for $\lambda$ positive and sufficiently small, and that a similar result holds for $\lambda<0$; moreover, the computer simulation is in complete agreement with this conjecture. In order to rigorously prove this claim, however, we should investigate more accurately the properties of the equation (3.36), trying to show that $\mathbf{T}^{n} v^{(\lambda)} \in \mathfrak{D}$ for any $\lambda$ sufficiently small, if $n$ is large enough. We think that this is possible, but we did not try to fill in the details.

\section{The correlation functions}

Let us suppose that $\varepsilon, \eta$ and $d$ are chosen so that there is in $\mathfrak{D}$ a fixed point $v^{*}$ of the transformation $\mathbf{T}$. We want to study the linear operator $\mathbf{L}_{v}$, see (2.13), for $v=v^{*}$; let us simply call it $\mathbf{L}$.

We shall consider the action of $\mathbf{L}$ on the Hilbert space $\mathfrak{H}$ of the functions periodic of period $T_{\alpha}=2 \pi / \alpha$ with inner product

$$
(G, F)=\frac{1}{T_{\alpha}} \int_{0}^{T_{\alpha}} d \varphi q(\varphi) G^{*}(\varphi) F(\varphi)
$$

where

$$
q(\varphi)=e^{v(\varphi)} \int P_{0}(d z) e^{v(\varphi+z)} \equiv e^{v(\varphi)} N(\varphi)
$$

Proposition 4.1 L is a trace class positive self-adjoint operator of norm 1 . 
Proof - It is very easy to verify that $\mathbf{L}$ is self-adjoint, by using the fact that the measure $P_{0}(d z)$ is even in $z$.

Let us now observe that the functions

$$
\psi_{Q}(\varphi)=q(\varphi)^{-\frac{1}{2}} e^{i \alpha Q \varphi} \quad, \quad Q \in \mathbb{Z}
$$

are a base of $\mathfrak{H}$ and that we can write:

$$
e^{v(\varphi)} q(\varphi)^{-\frac{1}{2}}=\sum_{Q} g_{Q} e^{i \alpha Q \varphi} \quad, \quad \sum_{Q}\left|g_{Q}\right|^{2}<\infty
$$

Then we have:

$$
\begin{aligned}
\operatorname{Tr} & (\mathbf{L})=\sum_{Q}\left(\psi_{Q}, \mathbf{L} \psi_{Q}\right)= \\
& =\sum_{Q} \int P_{0}(d z) \frac{1}{T_{\alpha}} \int_{0}^{T_{\alpha}} d \varphi\left[e^{v(\varphi)} \psi_{Q}^{*}(\varphi)\right]\left[e^{v(\varphi+z)} \psi_{Q}(\varphi+z)\right]= \\
& =\sum_{Q, Q^{\prime}}\left|g_{Q^{\prime}-Q}\right|^{2} \int P_{0}(d z) e^{i \alpha Q^{\prime} z}=\sum_{Q, Q^{\prime}}\left|g_{Q^{\prime}-Q}\right|^{2} \gamma^{-\frac{\alpha^{2}}{4 \pi} Q^{\prime 2}}= \\
& =\left(\sum_{Q}\left|g_{Q}\right|^{2}\right)\left(\sum_{Q^{\prime}} \gamma^{-\frac{\alpha^{2}}{4 \pi} Q^{\prime 2}}\right)<\infty
\end{aligned}
$$

which proves that $\mathbf{L}$ is trace class.

If $F \in \mathfrak{H}, e^{v} F \in \mathcal{L}_{2}$ and therefore we can write

$$
e^{v(\varphi)} F(\varphi)=\sum_{Q} \tilde{f}_{Q} e^{i \alpha Q \varphi} \quad, \quad \sum_{Q}\left|\tilde{f}_{Q}\right|^{2}<\infty
$$

Then, by proceeding as before, we can check that

$$
(F, \mathbf{L} F)=\sum_{Q}\left|\tilde{f}_{Q}\right|^{2} \gamma^{-\frac{\alpha^{2}}{4 \pi} Q^{2}}
$$

which proves that $\mathbf{L}$ is a positive operator. Moreover

$$
\begin{aligned}
& (F, \mathbf{L} F)=|(F, \mathbf{L} F)| \leq \\
& \leq \int P_{0}(d z) \frac{1}{2 T_{\alpha}} \int_{0}^{T_{\alpha}} d \varphi e^{v(\varphi)+v(\varphi+z)}\left[|F(\varphi)|^{2}+|F(\varphi+z)|^{2}\right]= \\
& =\frac{1}{T_{\alpha}} \int_{0}^{T_{\alpha}} d \varphi e^{v(\varphi)}|F(\varphi)|^{2} N_{v}(\varphi)=(F, F)
\end{aligned}
$$


Since $\mathbf{L} F=F$, if $F$ is a constant function, $\|\mathbf{L}\|=1$.

By Prop. $4.1 \mathbf{L}$ is a positive compact operator, then it has a pure discrete point spectrum with positive eigenvalues, at most finitely degenerate. Furthermore the subspaces $\mathfrak{H}^{+}$and $\mathfrak{H}^{-}$of $\mathfrak{H}$, which contain the functions even and odd in $\varphi$ respectively, are invariant under the action of $\mathbf{L}$. Let $\mathbf{L}^{ \pm}$be the restriction of $\mathbf{L}$ to $\mathfrak{H}^{ \pm}$.

For $\varepsilon=0$ (and hence $v=0$ ) the eigenvalues of $\mathbf{L}^{+}$and $\mathbf{L}^{-}$are the same, that is:

$$
\lambda_{n}^{ \pm}(0)=\gamma^{-2 n^{2}} \quad, \quad n=0,1, \ldots
$$

and they are all simple. By using the properties of $v$ proven in Sect. 3 and known results about the perturbation theory of compact operators, it is possible to show that the eigenvalues of $\mathbf{L}^{ \pm}$can be written as suitable functions $\lambda_{n}^{ \pm}(\varepsilon)$, which are continuous in $\varepsilon=0$.

Since the constants are eigenfunctions of $\mathbf{L}$ for any $\varepsilon \leq \varepsilon_{1}$ :

$$
\lambda_{0}^{+}=\lambda_{0}^{-}=1
$$

and 1 is a simple eigenvalue of $\mathbf{L}$. All the other eigenvalues are strictly less than 1 .

Let $\left\{\mu_{n}\right\}_{n \geq 0}$ be the set of all eigenvalues, ordered so that $\mu_{n+1} \leq \mu_{n}$, and let $G_{n}$ the corresponding eigenfunctions, normalized so that $G_{n}$ is real and

$$
\left(G_{n}, G_{m}\right)=\delta_{n m}
$$

In particular $\mu_{0}=1$ and

$$
G_{0}=\left[\frac{1}{T_{\alpha}} \int_{0}^{T_{\alpha}} d \varphi q(\varphi)\right]^{-\frac{1}{2}}
$$

Moreover, it is possible to show, by the technique used below in the proof of Theorem 4.1, that the $G_{n}$ are smooth functions.

Let us consider a function $F \in \mathfrak{H}$ such that its expansion in terms of the $G_{n}$ :

$$
F(\varphi)=\sum_{n=0}^{\infty} f_{n} G_{n}(\varphi)
$$


has good convergence properties. Then, by (2.11) we have:

$$
\begin{aligned}
< & F\left(\varphi_{0}\right)>_{v}=<\left(\mathbf{L}^{k} F\right)\left(\varphi_{0}\right)>_{v}= \\
& =\sum_{n} f_{n} \mu_{n}^{k}<G_{n}\left(\varphi_{0}\right)>_{v} \underset{k \rightarrow \infty}{\longrightarrow} f_{0} G_{0}=\left(G_{0}, F\right) G_{0}
\end{aligned}
$$

Let us now suppose that we want to calculate the correlation between $F\left(\varphi_{0}\right)$ and $F^{*}\left(\varphi_{x}\right)$. This problem arises, for example, if we are interested in the two charges correlation; in this case $F(\varphi)=e^{i \alpha \varphi}$, whose expansion has the needed convergence properties, as it is possible to show with some standard calculation, using the smoothness of the functions $G_{n}$ and the fact that they are small perturbations of the functions $e^{i \alpha Q}$.

If $h$ is the smallest integer so that there exists a $\Delta \in Q_{h}$ containing both 0 and $x$, we can write:

$$
\begin{gathered}
<F\left(\varphi_{0}\right) F^{*}\left(\varphi_{x}\right)>_{v}=<\left|\left(\mathbf{T}^{h} F\right)\left(\varphi_{0}\right)\right|^{2}>_{v}= \\
=\sum_{n m} f_{n} f_{m} \mu_{n}^{h} \mu_{m}^{h}<G_{n}\left(\varphi_{0}\right) G_{m}\left(\varphi_{0}\right)>_{v}
\end{gathered}
$$

But, by (4.11) and (4.14):

$$
<G_{n}\left(\varphi_{0}\right) G_{m}\left(\varphi_{0}\right)>_{v}=\left(G_{0}, G_{n} G_{m}\right) G_{0}=G_{0}^{2}\left(G_{n}, G_{m}\right)=G_{0}^{2} \delta_{n m}
$$

Then

$$
\begin{aligned}
<F\left(\varphi_{0}\right) F^{*}\left(\varphi_{x}\right)>_{v}^{T} & =<F\left(\varphi_{0}\right) F^{*}\left(\varphi_{x}\right)>_{v}-\left|<F\left(\varphi_{0}\right)>_{v}\right|^{2}= \\
& =G_{0}^{2} \sum_{n=1}^{\infty} \mu_{n}^{2 h}\left|f_{n}\right|^{2}
\end{aligned}
$$

and, as a consequence

$$
<F\left(\varphi_{0}\right) F^{*}\left(\varphi_{x}\right)>_{v}^{T} \quad \stackrel{\simeq}{\rightarrow} c \mu_{1}^{2 h}=c d_{0 x}^{-\tau}
$$

with $c$ a suitable constant and

$$
\tau=-2 \log _{\gamma} \mu_{1}
$$

We now want to show that

$$
\mu_{1}=\gamma^{-2}
$$


at least for $\varepsilon$ small enough. We start by observing that, since $\mathbf{T} v=v$, by (2.12):

$$
e^{\frac{1}{\gamma^{2} v(\varphi)}}=\frac{\int P_{0}(d z) e^{v(\varphi+z)}}{\int P_{0}(d z) e^{v(z)}}
$$

By calculating the $\varphi$-derivative of both sides, it is easy to check that:

$$
\mathbf{L} \frac{d v}{d \varphi}=\gamma^{-2} \frac{d v}{d \varphi}
$$

Since $\frac{d v}{d \varphi} \in \mathfrak{H}^{-}$, this implies that

$$
\lambda_{1}^{-}(\varepsilon)=\gamma^{-2} \quad, \quad \forall \varepsilon \leq \varepsilon_{1}
$$

But $\mu_{1}$, for $\varepsilon$ small enough, is the minimum between $\lambda_{1}^{+}(\varepsilon)$ and $\lambda_{1}^{-}(\varepsilon)$; hence, in order to show (4.20) it is sufficient to prove that $\lambda_{1}^{+}(\varepsilon)$ is smaller than $\gamma^{-2}$.

We notice that $(2.13)$ can be written also in the following way:

$$
\left(\mathbf{L}_{v} F\right)(\varphi)=\left.\frac{d}{d \lambda} \log \int P_{0}(d z) e^{v(\varphi+z)+\lambda F(\varphi+z)}\right|_{\lambda=0}
$$

If $F \in \mathfrak{H}, F \in \mathcal{L}_{2}$, then we can write:

$$
F(\varphi)=\sum_{Q} f_{Q} e^{i \alpha Q \varphi}
$$

Moreover, since $\left(\mathbf{L}_{v} F\right)(\varphi)$ does not change if we add a constant to $v(\varphi)$, we can replace in (4.24) $v(\varphi)$ by the expansion $\sum_{Q \neq 0} v_{Q} e^{i \alpha Q \varphi}$, whose coefficients are the same as in (3.1). Then we obtain:

$$
\begin{aligned}
& \left(\mathbf{L}_{v} F\right)(\varphi)=\sum_{Q} f_{Q} \gamma^{-\frac{\alpha^{2}}{4 \pi} Q^{2}} e^{i \alpha Q \varphi}+ \\
& +\sum_{n=2}^{\infty} \frac{1}{(n-1) !} \sum_{Q_{1}, \ldots, Q_{n}} v_{Q_{1}} \cdots v_{Q_{n-1}} f_{Q_{n}} F_{n}\left(Q_{1}, \ldots, Q_{n}\right) e^{i \alpha\left(\sum_{i=1}^{n} Q_{i}\right) \varphi}
\end{aligned}
$$

If $\lambda<1$, the eigenvalue equation $\mathbf{L}_{v} F=\lambda F$ is satisfied if $f_{0}=0$ and

$$
\begin{aligned}
\lambda f_{Q} & =f_{Q} \gamma^{-\frac{\alpha^{2}}{4 \pi} Q^{2}}+ \\
& +\sum_{n=2}^{\infty} \frac{1}{(n-1) !} \sum_{Q_{1}+\cdots+Q_{n}=Q} v_{Q_{1}} \cdots v_{Q_{n-1}} f_{Q_{n}} F_{n}\left(Q_{1}, \ldots, Q_{n}\right)
\end{aligned}
$$


for $|Q| \neq 0$.

We are interested in the dependence on $\varepsilon$ of the eigenvalue $\lambda_{1}^{+}(\varepsilon)$ and of the Fourier coefficients $f_{Q}(\varepsilon)=f_{-Q}(\varepsilon)$ of the corresponding eigenfunction, which we shall normalize so that

$$
f_{1}(\varepsilon)=f_{-1}(\varepsilon)=1
$$

For $\varepsilon=0$, we have

$$
f_{1}(0)=f_{-1}(0)=1 \quad, \quad f_{Q}=0 \quad \text { if }|Q| \neq 1
$$

We shall now rewrite (4.27), for $\lambda=\lambda_{1}^{+}(\varepsilon)$, as a fixed point equation in a suitable Banach space, where the existence of a unique solution will follow from the contraction mapping principle.

Let us define:

$$
\begin{aligned}
& G_{Q}(F)=\sum_{n=2}^{\infty} \frac{1}{(n-1) !} . \\
& \quad \cdot \sum_{\left|Q^{\prime}\right| \geq 2} f_{Q^{\prime}} \sum_{Q_{1}+\cdots+Q_{n-1}=Q-Q^{\prime}} v_{Q_{1}} \cdots v_{Q_{n-1}} F_{n}\left(Q_{1}, \ldots, Q_{n-1}, Q^{\prime}\right)
\end{aligned}
$$

Eq. (4.27) gives, for $Q=1$ :

$$
\lambda_{1}^{+}(\varepsilon)=\gamma^{-2}+r(\varepsilon)+G_{1}(F)
$$

where

$$
\begin{aligned}
& r(\varepsilon)=\gamma^{-2}\left(\gamma^{\varepsilon}-1\right)+v_{2} F_{2}(2,-1)+\sum_{n=3}^{\infty} \frac{1}{(n-1) !} . \\
& \cdot \sum_{Q= \pm 1} \sum_{Q_{1}+\cdots+Q_{n-1}=1-Q} v_{Q_{1}} \cdots v_{Q_{n-1}} F_{n}\left(Q_{1}, \ldots, Q_{n-1}, Q\right)
\end{aligned}
$$

If $|Q| \geq 2$, we have:

$$
\left[\lambda_{1}^{+}(\varepsilon)-\gamma^{-\frac{\alpha^{2}}{4 \pi} Q^{2}}\right] f_{Q}=h_{Q}+G_{Q}(F)
$$

where

$$
h_{Q}=\sum_{n=2}^{\infty} \frac{1}{(n-1) !} \sum_{\left|Q^{\prime}\right|=1} \sum_{Q_{1}+\cdots+Q_{n-1}=Q-Q^{\prime}} v_{Q_{1}} \cdots v_{Q_{n-1}} F_{n}\left(Q_{1}, \ldots, Q_{n-1}, Q^{\prime}\right)
$$


By proceeding as in the proof of Theorem 3.1, it is easy to show that there exist constants $a_{1}$ and $A_{1}$, only depending on $\bar{\delta}$ and $\delta_{1}$, such that

$$
\begin{gathered}
|r(\varepsilon)| \leq a_{1} \varepsilon \\
\left|h_{Q}\right| \leq A_{1} d \delta^{|Q|-1} \quad, \quad \text { if }|Q| \geq 2
\end{gathered}
$$

Let us now consider the Banach space $\mathfrak{E}$ of the even functions $F(\varphi)$, periodic of period $T_{\alpha}$, such that $f_{0}=0, f_{1}=f_{-1}=1$ and

$$
\|F\| \equiv \sup _{Q \geq 2} \delta^{-Q+1}\left|f_{Q}\right|<\infty
$$

We denote $\mathfrak{E}_{D}$ the sphere of radius $D$ and center at the origin and we consider the operator $\mathbf{K}$ from $\mathfrak{E}_{D}$ to $\mathfrak{H}_{+}$, defined so that, if $(\mathbf{K} F)(\varphi)=\sum_{Q \neq 0} f_{Q}^{\prime} e^{i \alpha Q \varphi}$, then

$$
\begin{aligned}
f_{Q}^{\prime} & =f_{-Q}^{\prime}=\frac{h_{Q}+G_{Q}(F)}{\gamma^{-2}+r(\varepsilon)+G_{1}(F)-\gamma^{-\frac{\alpha^{2}}{4 \pi} Q^{2}}} \quad, \quad \text { if } Q \geq 2 \\
f_{1}^{\prime}=f_{-1}^{\prime} & =1 \quad, \quad f_{0}^{\prime}=0
\end{aligned}
$$

By (4.31) and (4.33) $F$ is a solution of (4.27) for $\lambda=\lambda_{1}^{+}(\varepsilon)$, belonging to $\mathfrak{E}_{D}$, if and only if $F$ is a fixed point of the operator $\mathbf{K}$.

Theorem 4.1 There exist a positive constant $d_{2} \leq d_{1}$ and, for any given $d \leq d_{2}$ and $0<\eta<1 / 2$, other constants $\varepsilon_{2}, D_{0}$ and $D_{1}$, such that $\mathfrak{E}_{D}$ is invariant under the transformation $\mathbf{K}$, if $\varepsilon \leq \varepsilon_{2}$ and $D_{0} \leq D \leq D_{1}$; moreover $\mathbf{K}$ is a contraction as an operator from $\mathfrak{E}_{D}$ to $\mathfrak{E}_{D}$.

Proof - By proceeding as in the proof of Theorem 3.1, it is possible to show that, if $Q \geq 2$ and $D d$ is small enough:

$$
\left|f_{Q}^{\prime}\right| \leq \frac{A_{1} d(1+D) \delta^{Q-1}}{\gamma^{-2}-a_{1} \varepsilon-D d A_{1} \delta-\gamma^{-\frac{\alpha^{2}}{\pi}}}
$$

Therefore $\mathfrak{E}_{D}$ is invariant under the transformation $\mathbf{K}$ if

$$
D \geq \frac{A_{1} d(1+D)}{\gamma^{-2}-a_{1} \varepsilon-D d A_{1} \delta-\gamma^{-\frac{\alpha^{2}}{\pi}}}
$$


and it is easy to see that there exist $d_{2}, \varepsilon_{2}, D_{0}$ and $D_{1}$ such that (4.40) is satisfied if $d \leq d_{2}, \varepsilon \leq \varepsilon_{2}$ and $D_{0} \leq D \leq D_{1}$.

Let us now consider two elements $F_{1}, F_{2} \in \mathfrak{E}_{D}$ such that, for $Q \geq 2$,

$$
\left|f_{1 Q}-f_{2 Q}\right| \leq \rho \delta^{Q-1}
$$

It is easy to check that, for any $Q \geq 1$ :

$$
\left|G_{Q}\left(F_{1}\right)-G_{Q}\left(F_{2}\right)\right| \leq \rho d A_{1} \delta^{Q-1}
$$

Moreover, if $Q \geq 2$, by (4.38):

$$
\begin{aligned}
& f_{1 Q}^{\prime}-f_{2 Q}^{\prime}=\left\{h_{Q}\left[G_{1}\left(F_{2}\right)-G_{1}\left(F_{1}\right)\right]+b_{Q}\left[G_{Q}\left(F_{1}\right)-G_{Q}\left(F_{2}\right)\right]+\right. \\
& \left.\quad+G_{Q}\left(F_{1}\right)\left[G_{1}\left(F_{2}\right)-G_{1}\left(F_{1}\right)\right]+G_{1}\left(F_{1}\right)\left[G_{Q}\left(F_{1}\right)-G_{Q}\left(F_{2}\right)\right]\right\} / \\
& \quad /\left\{\left[b_{Q}+G_{1}\left(F_{1}\right)\right]\left[b_{Q}+G_{1}\left(F_{2}\right)\right]\right\}
\end{aligned}
$$

where

$$
b_{Q}=r(\varepsilon)+\gamma^{-2}-\gamma^{-\frac{\alpha^{2}}{4 \pi} Q^{2}}
$$

Then it is very easy to show that, for $d$ small enough,

$$
\left|f_{1 Q}^{\prime}-f_{2 Q}^{\prime}\right| \leq \bar{\alpha} \rho \quad, \quad \bar{\alpha}<1
$$

which immediately implies, together with Theorem 3.1, all the claims of this theorem.

From (4.30), (4.31), (4.32), Theorem 4.1 and some simple algebra follows that:

$$
\lambda_{1}^{+}(\varepsilon)=\gamma^{-2}-\varepsilon \log \gamma\left(1+\frac{a b}{a b+f(1-c)}\right)+O\left(\varepsilon^{3 / 2}\right)
$$

Then, if $\varepsilon$ is small enough

$$
\lambda_{1}^{+}(\varepsilon)<\lambda_{1}^{-}(\varepsilon)
$$

so that (4.20) is satisfied.

This means that, if $v=v^{*}$, the integer charge truncated correlations decay as $d_{x y}^{-4}$. With some more computational effort one could show that this result is true also if $v$ is in the domain of attraction of $v^{*}$.

We conclude by two remarks. The first remark, anticipated in the discussion preceding (4.13), is that the technique used in this section can be 
applied to any eigenvalue of $\mathbf{L}_{v}$ with similar results. In particular, one can show that, for any given $n$ and $\varepsilon$ small enough (how small depends on $n$ ), $\lambda_{n}^{ \pm}(\varepsilon)<\lambda_{n}^{ \pm}(0)$.

The second remark is that we could study the fractional charges correlations, by using similar arguments, in spite of the fact that the function $e^{i \alpha \varphi}$ must be substituted by $e^{i \alpha \xi \varphi}, 0<\xi<1$, which is not periodic of period $T_{\alpha}$. It is only sufficient to observe that, if $\bar{F}(\varphi)=e^{i \alpha \xi \varphi} F(\varphi)$, with $F \in \mathfrak{H}$, then

$$
\begin{gathered}
\left(\mathbf{L}_{v} \bar{F}\right)(\varphi)=e^{i \alpha \xi \varphi}\left(\mathbf{L}_{v}^{(\xi)} F\right)(\varphi) \\
\left(\mathbf{L}_{v}^{(\xi)} F\right)(\varphi)=\frac{\int P_{0}(d z) e^{i \alpha \xi \varphi} e^{v(\varphi+z)} F(\varphi+z)}{\int P_{0}(d z) e^{v(\varphi+z)}}
\end{gathered}
$$

and $\mathbf{L}_{v}^{(\xi)}$ is again a self-adjoint operator from $\mathfrak{H}$ to $\mathfrak{H}$, whose spectrum can be studied in the same way as the spectrum of $\mathbf{L}_{v}$, obtaining the same results reported in Ref. [6].

\section{Acknowledgements.}

We are indebted to G. Gallavotti and F. Nicolò for many discussions and suggestions.

\section{A Proof of the bound (3.4)}

Let $I=\{1, \ldots, n\}$ be the set of the first $n$ positive integers and, for each $i \in I$, let $Q_{i}$ be a fixed integer $\left(Q_{i} \in \mathbf{Z}\right)$. If $z_{t}$ is a random gaussian variable with mean 0 and covariance

$$
\mathcal{E}\left(z_{t}^{2}\right)=t \leq 1
$$

and $c$ is a fixed positive constant, we define:

$$
F(I, t)=\mathcal{E}^{T}\left(e^{i \sqrt{c} Q_{1} z_{t}} ; \ldots ; e^{i \sqrt{c} Q_{n} z_{t}}\right)
$$

where $\mathcal{E}^{T}$ denotes the truncated expectation with respect to $z_{t}$. It is a well known fact that:

$$
F(I, t)=e^{-\frac{c}{2} \sum_{i=1}^{n} Q_{i}^{2} t} f(I, t)
$$


with

$$
f(I, t)=\sum_{G} \prod_{i j \in G}\left(e^{-c Q_{i} Q_{j} t}-1\right)
$$

where $G$ is the family of all connected graphs on $n$ vertices labeled by the elements of $I$, with bonds denoted by $i j(i, j \in I)$.

Using the results of Ref. [9], in particular Lemma 3.3 and the recurrence relation for $f(I, t)$ which follows from it (see pag. 40 of [9]), it is very easy to show that:

$$
F(I, t)= \begin{cases}-\frac{c}{2} \int_{0}^{t} d s e^{-\frac{c}{2} Q_{I}^{2}(t-s)} \sum_{\emptyset \neq J \$ I} Q_{J} Q_{I \backslash J} F(J, s) F(I \backslash J, s) \\ e^{-\frac{c}{2} Q_{I}^{2} t} & \text { if }|I|=n \geq 2\end{cases}
$$

where we used the definition, for $J$ a subset of $I$ :

$$
Q_{J}=\sum_{i \in J} Q_{i}
$$

We want now to describe the solution of the recurrence relation (A5). Let us consider, for $n \geq 2$, the family $\Gamma_{n}$ of all planar binary trees with root $r$ and $n$ endpoints labeled by the elements of $I$, oriented from the root to the endpoints, see Fig. 1.

We call vertices the root, the endpoints (e.p. in the following) and the branch points of the tree; the branch points will be called also nontrivial (n.t. in the following) vertices. If $v$ is a vertex different from $r$, we shall denote by $v^{\prime}$ the vertex immediately preceding it in the tree and we shall say that $i \in v$ if the e.p. with label $i$ follows $v$; moreover $v_{0}$ will denote the vertex immediately following the root. We define:

$$
Q_{v}=\sum_{i \in v} Q_{i}
$$

Finally we label each vertex $v$ with a real number $s_{v}$ such that:

$$
\begin{gathered}
t \geq s_{v^{\prime}} \geq s_{v} \geq 0 \\
s_{r}=t \\
s_{v}=0 \quad \text { if } v \text { is an e.p. }
\end{gathered}
$$




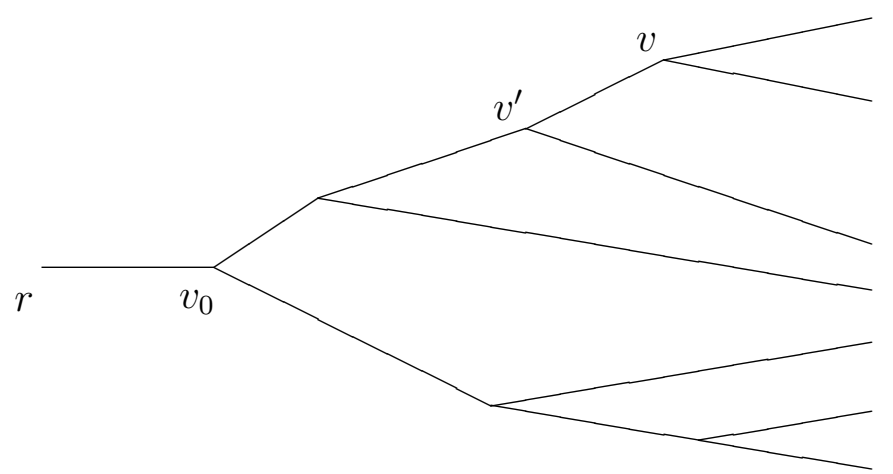

Fig. 1

It is easy to see that, if $|I| \geq 2$ :

$$
F(I, t)=\sum_{\theta \in \Gamma_{n}} \int\left(\prod_{v n . t .} \frac{-d s_{v}}{2}\right) \chi_{\theta} W_{\theta}
$$

where $\chi_{\theta}$ is the characteristic function of the set (A8) and

$$
\begin{aligned}
W_{\theta}= & \prod_{i=1}^{n}\left(\sqrt{c} Q_{i}\right)\left(\prod_{v e . p .} e^{-\frac{c}{2} Q_{i}^{2} s_{v^{\prime}}}\right) \cdot \\
\cdot & {\left[\prod_{\substack{v \text { n.t. } \\
v \neq v_{0}}}\left(\sqrt{c} Q_{v}\right) e^{-\frac{c}{2} Q_{v}^{2}\left(s_{v^{\prime}}-s_{v}\right)}\right] e^{-\frac{c}{2} Q_{I}^{2}\left(t-s_{v_{0}}\right)} }
\end{aligned}
$$

We want to show that

$$
|F(I, t)| \leq n^{n-1} \prod_{i=1}^{n}\left(2 \sqrt{c}\left|Q_{i}\right|\right) e^{-\frac{c}{4 n} Q_{I}^{2} t}
$$

The first step in the proof is to get rid of the "bad" factors $Q_{v}$ in (A10), using the bound

$$
\sqrt{c}\left|Q_{v}\right| e^{-\frac{c}{2} Q_{v}^{2}\left(s_{v^{\prime}}-s_{v}\right)} \leq \frac{1}{\sqrt{s_{v^{\prime}}-s_{v}}} e^{-\frac{c}{4} Q_{v}^{2}\left(s_{v^{\prime}}-s_{v}\right)}
$$


Then, if $|I| \geq 2$, we can write, using also that $0 \leq t-s_{v_{0}} \leq 1$ :

$$
|F(I, t)| \leq \prod_{i=1}^{n}\left(\sqrt{c}\left|Q_{i}\right|\right) E(I, t)
$$

where

$$
E(I, t)= \begin{cases}\frac{1}{2} \int_{0}^{t} \frac{d s}{\sqrt{t-s}} e^{-\frac{c}{4} Q_{I}^{2}(t-s)} \sum_{\emptyset \neq J \$ I} E(J, s) E(I \backslash J, s) \\ e^{-\frac{c}{2} Q_{I}^{2} t} & \text { if }|I|=n \geq 2\end{cases}
$$

We now prove, by induction on $n=|I|$, that:

$$
E(I, t) \leq(2 n)^{n-1} e^{-\frac{c}{4 n} Q_{I}^{2} t}
$$

In fact (A15) is true for $n=1$; moreover, if we suppose that it is true for $1 \leq k<n$, we have, using (A14):

$$
E(I, t) \leq 2^{n-3} \sum_{\emptyset \neq J \$ I} k^{k-1}(n-k)^{n-k-1} G(I, J, t)
$$

where $k=|J|$ and

$$
G(I, J, t)=e^{-\frac{c}{4} Q_{I}^{2} t} \int_{0}^{t} \frac{d s}{\sqrt{t-s}} e^{\frac{c}{4} s\left[Q_{I}^{2}-Q_{J}^{2} / k-Q_{I \backslash J}^{2} /(n-k)\right]}
$$

If $\left[Q_{I}^{2}-Q_{J}^{2} / k-Q_{I \backslash J}^{2} /(n-k)\right] \geq 0$, then

$$
G(I, J, t) \leq e^{-\frac{c}{4} t\left[Q_{J}^{2} / k+Q_{I \backslash J}^{2} /(n-k)\right]} \int_{0}^{t} \frac{d s}{\sqrt{t-s}} \leq 2 e^{-\frac{c}{4 n} t Q_{I}^{2}}
$$

where we used the fact that $t \leq 1$ and the inequality, valid for two arbitrary real numbers $a$ and $b$ :

$$
\frac{a^{2}}{k}+\frac{b^{2}}{n-k} \geq \frac{1}{n}(a+b)^{2}
$$

If $\left[Q_{I}^{2}-Q_{J}^{2} / k-Q_{I \backslash J}^{2} /(n-k)\right] \leq 0$, then

$$
G(I, J, t) \leq e^{-\frac{c}{4} t Q_{I}^{2}} \int_{0}^{t} \frac{d s}{\sqrt{t-s}} \leq 2 e^{-\frac{c}{4 n} t Q_{I}^{2}}
$$


(A16), (A18) and (A20) imply that:

$$
E(I, t) \leq 2^{n-2} e^{-\frac{c}{4 n} Q_{I}^{2} t} \sum_{k=1}^{n-1}\left(\begin{array}{l}
n \\
k
\end{array}\right) k^{k-1}(n-k)^{n-k-1} \leq(2 n)^{n-1} e^{-\frac{c}{4 n} Q_{I}^{2} t}
$$

where we used the identity (see [9]: Lemma 4.2):

$$
\sum_{k=1}^{n-1}\left(\begin{array}{l}
n \\
k
\end{array}\right) k^{k-1}(n-k)^{n-k-1}=2(n-1) n^{n-2}
$$

Then (A15) is proved; if we insert it into (A13), we obtain the bound (A11).

\section{References}

[1] J.M. Kosterlitz and D.J. Thouless. Ordering, metastability and phase transitions in two-dimensional systems. J. Phys., C6:1181-1203, 1973.

[2] J. Fröhlich and T. Spencer. The Kosterlitz-Thouless transition in two dimension Abelian spin systems and the Coulomb gas. Commun. Math. Phys., 81:527-602, 1981.

[3] D.H.U. Marchetti, A. Klein, and J. Fernando Perez. Power law fall off in the Kosterlitz-Thouless phase of a two-dimensional lattice Coulomb gas. J. Stat. Phys., 60:137, 1990.

[4] J. Dimock and T. R. Hurd. A renormalization group analysis of the Kosterlitz-Thouless phase. Commun. Math. Phys., 137:263-287, 1991.

[5] G. Benfatto, G. Gallavotti, and F. Nicolò. The dipole phase in two dimensional hierarchical Coulomb gas: analyticity and correlations decay. Commun. Math. Phys., 106:227-288, 1986.

[6] D.H.U. Marchetti and J. Fernando Perez. The Kosterlitz-Thouless phase transition in two-dimensional hierarchical Coulomb gases. J. Stat. Phys., 55:141-156, 1989.

[7] D. Brydges and P. Federbush. Debye screening. Commun. Math. Phys., 73:197-246, 1980. 
[8] K. Gawedski and A. Kupiainen. Asymptotic freedom beyond perturbation theory. In Les Houches Summer School, 1984.

[9] D. Brydges and T. Kennedy. Mayer expansions and the Hamilton-Jacobi equation. J. Stat. Phys., 48:19-49, 1987. 